\title{
Jones-matrix mapping of optically anisotropic fluorophores of molecular biological tissues in the diagnostics of death causes
}

\author{
A.G. Ushenko ${ }^{1}$, A.V. Dubolazov ${ }^{1}$, Yu.A. Ushenko ${ }^{1}$, L.Ya. Kushnerick ${ }^{1}$, M.Yu. Sakhnovskiy ${ }^{1}$, V.G. Zhytaryuk ${ }^{1}$, \\ I. Lacusta ${ }^{1}$, O.G. Prydiy ${ }^{1}$, P. Grygorishin ${ }^{2}$ \\ ${ }^{1}$ Chernivtsi National University, \\ 2, Kotsyubinsky str., 58012 Chernivtsi, Ukraine \\ ${ }^{2}$ Bukovinian State Medical University, \\ 58000 Chernivtsi, Ukraine; \\ E-mail: yuriyu@gmail.com
}

\begin{abstract}
A model of generalized optical anisotropy of polycrystalline networks formed by albumin and globulin in human brain liquor has been suggested. The polarizationphase method of spatial and frequency differentiation of linear and circular birefringence coordinate distributions have been analytically substantiated. A set of criteria characterizing the dynamics of changes in polarization-phase images corresponding to necrotic changes in liquor polycrystalline films for determination of death coming prescription has been detected and substantiated.
\end{abstract}

Keywords: polarization, Fourier optics, signal processing, imaging systems, medical and biological imaging.

Manuscript received 11.10.16; revised version received 26.01.17; accepted for publication 01.03.17; published online 05.04.17.

\section{Principles of optical detection of death coming time}

\subsection{Introduction}

Among diverse optical-physical methods [1-8] of diagnosing the optical-anisotropic components in biological objects, a specific trend has been separated laser polarimetry [9-17] of microscopic images of biological tissues in approximation of linear birefringence of polycrystalline protein networks. At the same time, apart from linear birefringence, the mechanisms of transforming the state of laser radiation polarization by optical-anisotropic biological structures are more diverse and include optical activity or circular birefringence [7, 8]. The laser polarimetry techniques characterize general manifestations of these mechanisms in the process of polarizationally inhomogeneous images formation $[9,10]$. At the same time, each of these partial mechanisms is closely related with transformation of biochemical components of the biological layer [12, 13, $15,17]$. Consequently, pathological or necrotic changes are inevitably accompanied by the changes in various types of optical anisotropy. Thus, the task of optical differentiation between these mechanisms is important for development of laser polarimetry techniques in the task of determination of death coming prescription (DCP). At present time, forensic medicine needs essential renewal of the techniques of DCP estimation. It is related with the fact that existing methods depend on multiple factors of external environment and circumstances of death [18-32]. Existing lack of modern, objective techniques of DCP estimation stimulates the search and development of novel techniques for investigation of postmortem changes in human biological tissues and fluids [23]. Noninvasive optical methods of diagnostics of the structure of biological layers are perspective in this field. On this base, opening up the possibilities of objective and more precise estimation of interval from the moment of human death coming becomes real. Fourier analysis of polarizationally inhomogeneous images of biological layers can become one of possible solutions of this task 
$[24,25]$. The main idea of such an approach is based on the existence of differences in spatial-frequency spectra of Fourier images corresponding to non-uniformly scaled biological crystals with various anisotropy mechanisms.

Our research is aimed at designing an experimental method of Fourier laser polarization phasometry of the layers of human liquor for determination of death coming prescription by means of statistical analysis of time dynamics in the change of polarizationally filtered field of the scattered coherent radiation.

\subsection{The theory of method}

Conventional for medical practice optically thin (attenuation coefficient $\tau \approx 0.093 \ldots 0.096$ ) smears of human liquor on the homogeneous glass (refraction index $n=1.47$ ) dried at room temperature were used as objects of investigation.

The view human liquor is a phase-inhomogeneous fluid consisting of:

- large-scale $(100 \ldots 150 \mu \mathrm{m})$ acicular crystals of albumin with prevailing linear birefringence. Optical anisotropy of acicular structures is characterized by the following Jones matrix [16]

$$
\{G\}=\left\|\begin{array}{ll}
\left.\sin ^{2} \rho+\cos ^{2} \rho \exp (-i \omega)\right]_{11} & {[\sin \rho \cos \rho(1-\exp (-i \omega))]_{12}} \\
{[\sin \rho \cos \rho(1-\exp (-i \omega))]_{12}} & {\left[\cos ^{2} \rho+\sin ^{2} \rho \exp (-i \omega)\right]_{22}}
\end{array}\right\|
$$

Here, $\rho$ is the direction of the optical axes of an acicular crystal, $\omega=(2 \pi / \lambda) \Delta n L-$ value of a phase shift between the orthogonal components of the amplitude of laser wave with the length $\lambda$, which passed the geometric path $L$ through the crystal with a linear birefringence $\Delta n$.

- small-scale $(5 . .20 \mu \mathrm{m})$ globulin crystals with prevailing circular birefringence or optical activity characterized by the matrix [16]

$$
\{C\}=\left\|\begin{array}{cc}
\cos \psi & \sin \psi \\
-\sin \psi & \cos \psi
\end{array}\right\|
$$

Here, $\psi$ is the rotation angle of the laser wave polarization plane as a result of optical activity (circular birefringence - occurrence of the phase shift between the left and right circularly polarized components of the amplitude) of the crystal.

While the laser radiation is passing through this layer, due to the total impact of various anisotropy mechanisms a polarizationally inhomogeneous image is formed

$$
\begin{aligned}
& \left\{\begin{array}{l}
U_{x}(\rho, \omega)=g_{11} U_{0 x}+g_{12} U_{0 y}, \\
U_{y}(\rho, \omega)=g_{21} U_{0 x}+g_{22} U_{0 y},
\end{array}\right. \\
& \left\{\begin{array}{l}
U_{x}(\psi)=c_{11} U_{0 x}+c_{12} U_{0 y}, \\
U_{y}(\psi)=c_{21} U_{0 x}+c_{22} U_{0 y} .
\end{array}\right. \\
& \text { Here, }\left(\begin{array}{l}
U_{0 x} \\
U_{0 y}
\end{array}\right) \text { and }\left\{\left(\begin{array}{l}
U_{x}(\rho, \omega) \\
U_{y}(\rho, \omega)
\end{array}\right),\left(\begin{array}{l}
U_{x}(\psi) \\
U_{y}(\psi)
\end{array}\right)\right. \text { are Jones }
\end{aligned}
$$

vectors of probing and object waves.

From the medical viewpoint, the task of "optical separation" of polarization manifestations of linear and circular birefringence of albumin and globulin crystals is topical $[10,14,16,17]$. The fact is that necrotic changes in human organism are accompanied by the decrease of concentration of optically active globulins in liquor and the destruction of polycrystalline network in albumin crystals. To realize this task, we applied the method of the spatial-frequency filtration of a polarizationally inhomogeneous field corresponding to laser radiation in the Fourier plane $[24,25]$. The main idea of this approach lies in the fact that spatial-frequency structure of the Fourier form describing the laser image of the liquor polycrystalline network is different for its large scale albumin and small scale protein structures. Therefore, through space-frequency filtering, one can mainly select either low-frequency (with prevailing linear birefringence) or high-frequency (predominantly optically active) components, which can be converted into corresponding "separated" laser images by means of the reverse Fourier transformation.

Thus, the spatial-frequency filtering enables to differentiate laser images of various conformational components of the polycrystalline network in the liquor smear data. For experimental acquisition of "phase" information from the obtained images, the Fourier scheme of filtering was joined with the well-known techniques of polarization filtering. [26].

To measure the phase shift $\delta$ distribution, the sample was located between two crossed quarter-wave filtered plates $\left(\left\{F_{1}\right\}\right.$ and $\left.\left\{F_{2}\right\}\right)$. Polarizers $\left(\left\{P_{1}\right\}\right.$ and $\left\{P_{2}\right\}$ ) were used, which transmission planes make the angles with axes of the highest rate $+45^{\circ}$ and $-45^{\circ}$. The amplitude $\hat{U}$ in each point $(x, y)$ of the laser image is defined from the following network equation

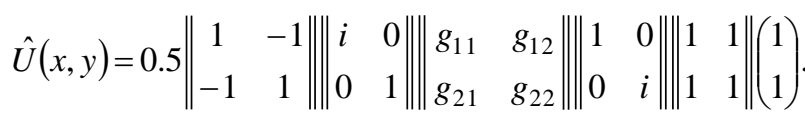

The following expressions are the solutions of Eq. (4)

$\omega(x, y)=2 \arcsin \sqrt{\frac{I_{\omega}(x, y)}{I_{0}}}$. 
Here, $I_{0}$ is the probing beam intensity, $I(\omega) \rightarrow\left\{\begin{array}{l}0 \leftrightarrow \omega=0, \\ 1 \leftrightarrow \omega=\pi\end{array} \quad-\quad\right.$ intensity of points of polarization-frequency filtered laser image.

Thus, to determine the distribution $\omega(x, y)$ in the "low-frequency" image of a polycrystalline network, it would be enough to register the array of normalized $\frac{I_{\omega}(x, y)}{I_{0}}$ intensity values by using a CCD-camera.

Similarly, to calculate the coordinate distribution $\psi(x, y)$ in the points of a "high-frequency" image of a polycrystalline network, the effusion smear was placed between two crossed plates $\left(\left\{P_{1}\right\}\right.$ and $\left\{P_{2}\right\}$ )

$\psi(x, y)=\arcsin 0.5 \sqrt{\frac{I_{\psi}(x, y)}{I_{0}}}$.

For an objective evaluation of distributions $v=\left\{\begin{array}{l}\omega(x, y) \\ \psi(x, y)\end{array}\right.$, the total sum of statistical moments of the $1^{\text {st }}$ to $4^{\text {-th }}$ orders $M_{j=1 ; 2 ; 3 ; 4}$ with the following algorithms was calculated [10]

$$
\begin{aligned}
& M_{1}=\frac{1}{N} \sum_{i=1}^{N}\left|(v)_{i}\right|, M_{2}=\sqrt{\frac{1}{N} \sum_{i=1}^{N}(v)_{i}^{2}} \\
& M_{3}=\frac{1}{\left(M_{2}\right)^{3}} \frac{1}{N} \sum_{i=1}^{N}(v)_{i}^{3}, M_{4}=\frac{1}{\left(M_{2}\right)^{4}} \frac{1}{N} \sum_{i=1}^{N}(v)_{i}^{4} .
\end{aligned}
$$

\section{Temporal dynamics of changing in the polarization-inhomogeneous images of liquor films}

\subsection{Optical scheme}

The optical scheme of Fourier polarimetry which was used in this work is described in details in [25].

The following two groups were used as objects of investigation in this research:

- intravital polycrystalline liquor films taken from donor-patients - Group 1 (48 samples);

- postmortal polycrystalline liquor films taken from the patients who died of myocardial infarction - Group 2 (48 samples).

Experimental study of time dynamics of postmortal change in biochemical structure of liquor films was performed using the following algorithm:

1. For each sample of polycrystalline liquor films, the coordinate distributions of phase-shift values were determined (relations (5), (6)).
2. The measurements of phase maps corresponding to polycrystalline liquor films were performed in two stages: $1^{\text {st }}$ - every $15 \mathrm{~min}$ for 6 hours from the moment of death; $2^{\text {nd }}-$ every hour up to 30 hours after the moment of death.

3. For each distribution of phase-shift values, the statistical moments of the $1^{\text {st }}$ to $4^{\text {th }}$ orders were calculated (relation (7)).

4. The time dependences of change in the statistical moments most sensitive to necrotic changes until the stabilization value of these parameters were determined.

\subsection{Spatial-frequency phase maps}

Fig. 1 presents phase maps (fragments (a), (b)) and distribution histograms (fragments (c), (d)) of random values $\delta(x, y)$ of the "low-frequency" component for the laser image of polycrystalline network of liquor smear at different time intervals from the death coming: 1 hour ((a), (c)) and 12 hours - ((b), (d)).

The comparative analysis of the aggregate parameters characterizing the phase maps of linear birefringence $\omega(m \times n)$ of large-scale optical anisotropic networks in liquor samples showed a sufficient difference between them. It is evident that the range of random phase values essentially decreases - practically by 2 times (fragments (b), (d)) - in 12 hours after death. Degenerative changes of polycrystalline structure in the liquor film are pronounced in destroying the highmolecular protein complexes possessing a high level of spatial orientation and correspondingly - noticeable birefringence in medium- and low-molecular structures with lower optical anisotropy.

It is quantitatively manifested in the decrease of the average and dispersion characterizing random phase values distributions of polycrystalline liquor films and the increase of observation time after the death moment. Statistical moments of higher orders (asymmetry and excess) of these polarizationally inhomogeneous images, on the contrary, increase.

Time monitoring of post-mortal changes of phase maps for liquor films proves the most dynamic change in the statistical moments of the $3^{\text {rd }}$ to $4^{\text {th }}$ orders (Table 1 ).

It goes from time monitoring the changes in the statistical structure of phase distributions corresponding to large-scale polycrystalline networks in liquor films that the interval of DCP determination $T=30$ hours.

Diagnostic possibilities of determination of death coming by using high-frequency Fourier phasometry of the rotation angle $\psi(x, y)$ illustrate probabilistic (fragments (c) - DCP $=1$ hour, $(d)-$ DCP $=6$ hours) dependences of the phase maps (fragments of (a), (b)) provided in Fig. 2. 


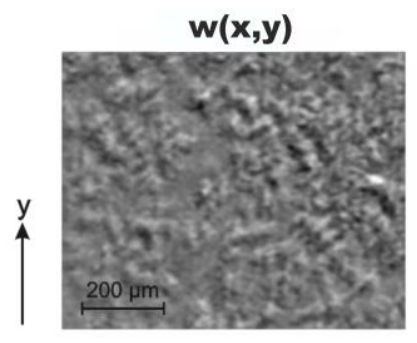

a)

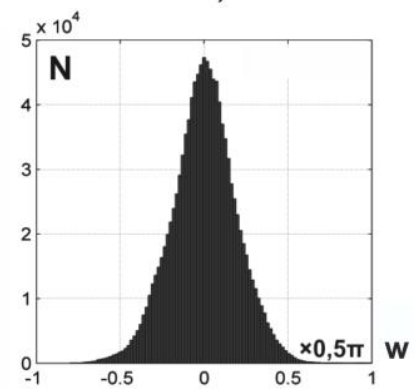

c)

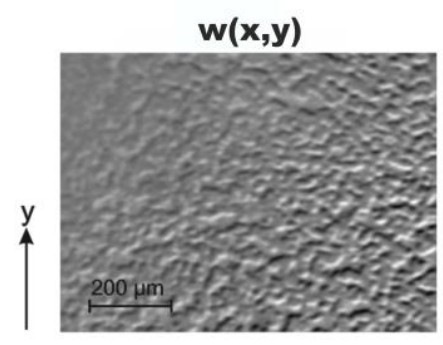

b)

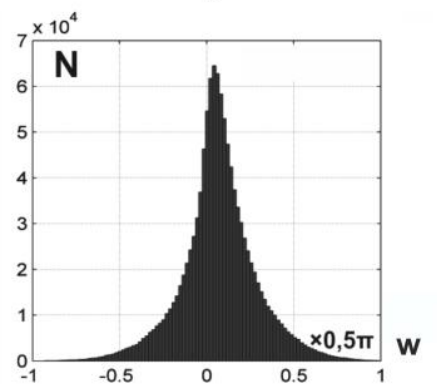

d)

Fig. 1. Phase maps (a), (b) and histograms (c), (d) of distribution corresponding to linear birefringence observed in liquor films.

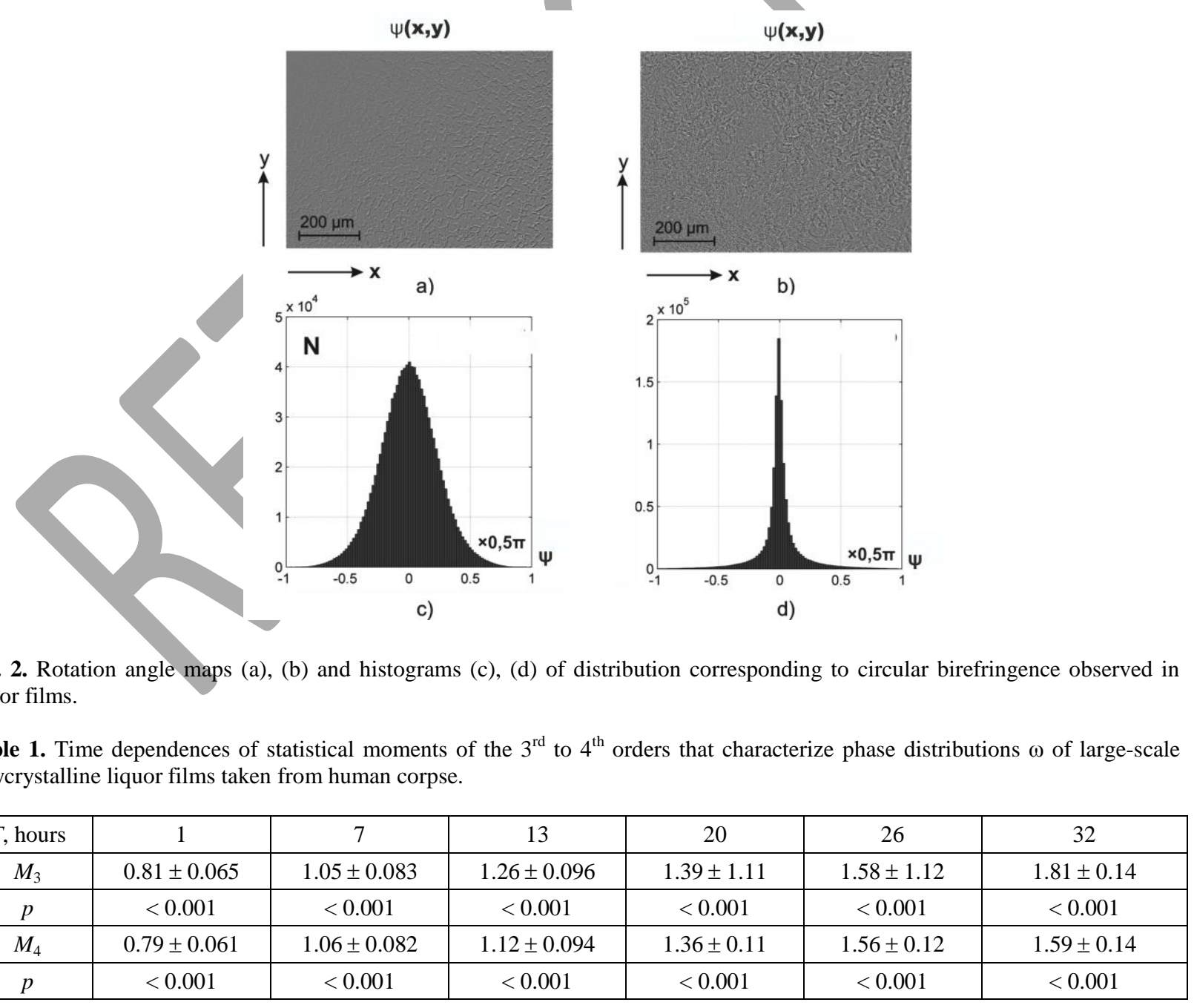


Table 2. Time dependences of statistical moments of the $3^{\text {rd }}$ to $4^{\text {th }}$ orders that characterize rotation angle distributions of the smallscale component in polycrystalline liquor films.

\begin{tabular}{|c|c|c|c|c|c|c|}
\hline$T$, hours & 1 & 3 & 5 & 7 & 10 & 14 \\
\hline$M_{3}$ & $1.09 \pm 0.085$ & $1.26 \pm 0.105$ & $1.43 \pm 0.11$ & $1.6 \pm 0.12$ & $1.77 \pm 0.14$ & $1.94 \pm 0.16$ \\
\hline$p$ & $<0.001$ & $<0.001$ & $<0.001$ & $<0.001$ & $<0.001$ & $<0.001$ \\
\hline$M_{4}$ & $1.06 \pm 0.086$ & $1.23 \pm 0.1$ & $1.4 \pm 0.11$ & $1.57 \pm 0.12$ & $1.74 \pm 0.14$ & $1.91 \pm 0.16$ \\
\hline$p$ & $<0.001$ & $<0.001$ & $<0.001$ & $<0.001$ & $<0.001$ & $<0.001$ \\
\hline
\end{tabular}

It is obvious that rotation angle maps of optically active molecular compounds represent the ensemble of small-scale zones, the amount of which essentially decreases with the DCP (death coming prescription) increase. This fact quantitatively characterizes the 4-fold decrease in the half-width range of random phase values in 6 hours after the moment of death. The observed degradation processes in the small-scale polycrystalline structure of liquor films can be related to the decrease in concentration and decay of chiral molecular complexes.

Table 2 presents the calculation results of statistical moments of the $3^{\text {rd }}$ to $4^{\text {th }}$ orders characterizing phase distributions of the small-scale component in globulin polycrystalline networks, liquor films glucose during 14 hours after death moment.

The comparative analysis with the data of largescale microscopic images revealed the decrease in the interval of DCP determination due to a more steep decrease of the values of statistical moments of the $3^{\text {rd }}$ to $4^{\text {th }}$ orders $T=14$ hours.

\subsection{Analytical algorithm for determining death coming prescription}

The following relations for DCP determining can be obtained from the analysis of the model scheme presented in Fig. 3.

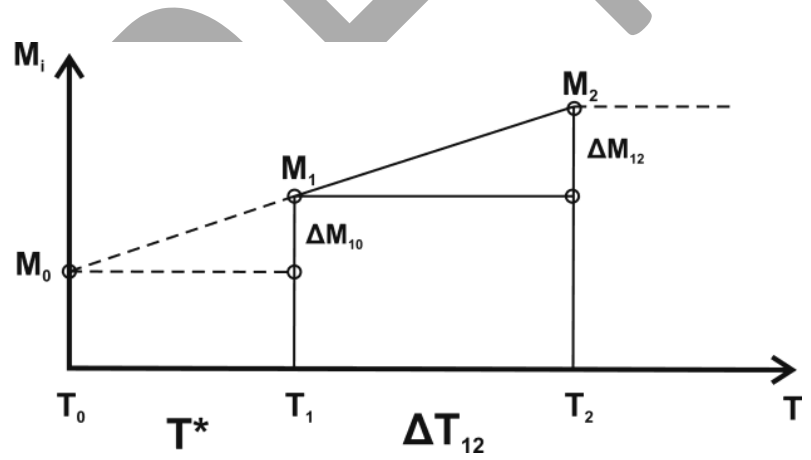

Fig. 3. To the analysis of the algorithm of DCP analytical determination. Here: $T_{1}$ is time of beginning the measurements of $M_{i}^{(1)} ; T_{2}$ - time of ending the measurement of $M_{i}^{(2)}$ at the stage of "stabilization" of its value change $\left(M_{i}^{(2)}(T) \approx\right.$ const $) ; T_{0}$ - time of death coming; $\zeta$ - inclination angle of information dependence $M_{i}(T)$.
From the geometric point of view, there is the following relationship between the values of the statistical moments and time intervals of their change

$$
\frac{\Delta M_{12}}{\Delta M_{01}}=\frac{\Delta T}{T^{*}}
$$

Using relation (8), the expression for DCP determination is obtained:

$$
T^{*}=\Delta T \frac{\Delta M_{01}}{\Delta M_{12}}
$$

Here, $M_{i}^{(0)}$ is the value of objective parameter determined by intravital liquor sampling from the donor (Table 3).

Table 3. Statistical moments of the $3^{\text {rd }}$ to $4^{\text {th }}$ orders characterizing phase maps for the intravital polycrystalline liquor films.

\begin{tabular}{|c|c|c|}
\hline$M_{i}^{0}$ & $\omega$ & $\psi$ \\
\hline$M_{3}^{0}$ & $0.57 \pm 0.039$ & $0.48 \pm 0.031$ \\
\hline$p$ & $<0.001$ & $<0.001$ \\
\hline$M_{4}^{0}$ & $0.44 \pm 0.034$ & $0.39 \pm 0.028$ \\
\hline$p$ & $<0.001$ & $<0.001$ \\
\hline
\end{tabular}

The following intervals and accuracy of DCP determination are presented in Table 4 .

Table 4. Intervals and accuracy of DCP determination.

\begin{tabular}{|l|c|c|}
\hline Parameters & $\begin{array}{c}\text { Interval of } \\
\text { DCP deter- } \\
\text { mination, } T\end{array}$ & $\begin{array}{c}\text { Accuracy of } \\
\text { DCP determi- } \\
\text { nation, } \Delta T\end{array}$ \\
\hline $\begin{array}{l}\text { Distribution of polari- } \\
\text { zation azimuths of the } \\
\text { large-scale component of } \\
\text { a microscopic image }\end{array}$ & 30 hours & 45 min \\
\hline $\begin{array}{l}\text { Distribution of polari- } \\
\text { zation azimuths of the } \\
\text { small-scale component of } \\
\text { a microscopic image }\end{array}$ & 14 hours & 25 min \\
\hline
\end{tabular}

\section{Conclusion}


The model of optical anisotropy for the liquor polycrystalline networks has been suggested, and the method of Fourier phasometry of linear parameters (phase shift between the orthogonal components of the laser wave amplitude) and circular (the angle of rotation of the polarization plane) birefringence with a spatialfrequency selection of the coordinate distributions for the death coming prescription determination have been analytically substantiated.

The hypothesis of linear changes of the values of statistical moments was confirmed by the $3^{\text {rd }}$ to $4^{\text {th }}$ orders characterizing phase distribution of different scale components in microscopic images of polycrystalline films.

The optical manifestations of polycrystalline networks of liquor, most sensitive to post-mortal changes - the statistical moments of the $3^{\text {rd }}$ to $4^{\text {th }}$ orders characterizing phase distributions of different scale components in microscopic images of polycrystalline films were detected.

The interval $T=30$ hours and accuracy $\Delta T=45$ min of DCP determination by means of the technique for determining phase distributions of the large-scale component in a microscopic image of liquor films was found.

The interval $T=14$ hours and accuracy $\Delta T=25$ min of DCP determination by means of the technique for determining phase distributions of the small-scale component in a microscopic image of liquor films was found.

\section{Acknowledgement}

This work was supported by the grant №0116U001446 №0116U001449, №0115U003241， №0115U003227, №0115U003235 from the Ukraine Foundation for Basic Research.

\section{References}

1. Tuchin V.V. Handbook of Coherent-Domain Optical Methods. Biomedical Diagnostics, Environmental and Material Science. Kluwer Academic Publishers, 2004.

2. Cheong W.-F., Prahl S.A., Welch A.J. A review of the optical properties of biological tissues. IEEE J. Quantum Electron. 1990. 26. P. 2166-2185.

3. Mishchenko M.I., Travis L.D., Lacis A.A. Scattering, Absorption and Emission of Light by Small Particles. Cambridge University Press, 2002.

4. Cowin S.C. How is a tissue built? J. Biomech. Eng. 2000. 122. P. 553-568.

5. de Boer J.F., Milner T.E., van Gemert M.J., Nelson J.S. Two-dimensional birefringence imaging in biological tissue using polarization-sensitive optical coherence tomography. Proc. SPIE. 1998. 3196. P. 32-37.

6. Everett M.J., Shoenenberger K., Colston B.W., Da Silva L.B. Birefringence characterization of biological tissue by use of optical coherence tomography. Opt. Lett. 1998. 23. P. 228-230.

7. de Boer J.F., Milner T.E., Nelson J.S. Determination of the depth-resolved Stokes parameters of light backscattered from turbid media by use of polarization-sensitive optical coherence tomography. Opt. Lett. 1999. 24. P. 300-302.

8. Angelsky O.V., Bekshaev A.Ya., Maksimyak P.P., Maksimyak A.P., Hanson S.G., Zenkova C.Yu. Self-diffraction of continuous laser radiation in a disperse medium with absorbing particles. Opt. Exp. 2013. 21, No. 7. P. 8922-8938.

9. Angelsky O.V., Bekshaev A.Ya., Maksimyak P.P., Maksimyak A.P., Hanson S.G. Measurement of small light absorption in microparticles by means of optically induced rotation. Opt. Exp. 2015. 23, No. 6. P. 7152-7163.

10. Angelsky O.V., Besaha R.N., Mokhun A.I., Mokhun I.I., Sopin M.O., Soskin M.S., Vasnetsov M.V. Singularities in vectoral fields. Proc. SPIE. 1999. 3904. P. 40.

11. Ushenko Yu.O., Tomka Yu.Ya., Telenga O.I., Misevitch I.Z., Istratiy V.V. Complex degree of mutual anisotropy of biological liquid crystals nets. Opt. Eng. 2011. 50. P. 039001.

12. Ushenko, A.G. Polarization correlometry of angular structure in the microrelief pattern of rough surfaces. Optics and Spectroscopy. 2002. 92. P. 227-229.

13. Polyanskii V.K., Angelsky O.V., Polyanskii P.V. Scattering-induced spectral changes as a singular optical effect. Optica Applicata. 2002. 32, No. 4. P. 843-848.

14. Ushenko V.A., Sidor M.I., Marchuk Y.F., Pashkovskaya N.V., Andreichuk D.R. Azimuthinvariant Mueller-matrix differentiation of the optical anisotropy of biological tissues. Optics and Spectroscopy. 2014. 117, No. 1. P. 152-157.

15. Ushenko V.A., Zabolotna N.I., Pavlov S.V., Burcovets D.M., Novakovska O.Yu. Muellermatrices polarization selection of two-dimensional linear and circular birefringence images. Proc. SPIE. 2013. 9066, Eleventh International Conference on Correlation Optics. P. 90661X.

16. Ushenko V.A., Gorsky M.P. Complex degree of mutual anisotropy of linear birefringence and optical activity of biological tissues in diagnostics of prostate cancer. Optics and Spectroscopy. 2013. 115, No. 2. P. 290-297.

17. Ushenko Yu.A. Concerted spatial-frequency and polarization-phase filtering of laser images of polycrystalline networks of blood plasma smears. $J$. Biomed. Opt. 2012. 17, No. 11. P. 117005.

18. Ushenko Yu.O., Dubolazov O.V., Karachevtsev A.O., Gorsky M.P., Marchuk Y.F. Wavelet analysis of Fourier polarized images of the human bile. Appl. Opt. 2012. 51, No. 10. P. C133-C139.

19. Ushenko Yu.A., Ushenko V.A., Dubolazov A.V., Balanetskaya V.O., Zabolotna N.I. Mueller-matrix diagnostics of optical properties of polycrystalline 
networks of human blood plasma. Optics and Spectroscopy, 2012. 112, No. 6. P. 884-892.

20. Ushenko Yu.A., Tomka Yu.Ya., Dubolazov A.V. Laser diagnostics of anisotropy in birefringent networks of biological tissues in different physiological conditions. Quantum Electronics. 2011. 41, No. 2. P. 170-175.

21. Ushenko Yu.A., Dubolazov A.V., Balanetskaya V.O., Karachevtsev A.O., Ushenko V.A. Waveletanalysis of polarization maps of human blood plasma. Optics and Spectroscopy. 2012. 113, No. 3. P. 332-343.

22. Joachim H., Feldmann U., Eine Quantitative Methode Der Todeszeitbestimmung Durch Untersuchung Der Galvanischen Reizschwelle (Rheobase) Am Skelettmuskel Von Leichen. Z. Rechtsmed. 1980. 85, No. 1. P. 5-22.

23. Kuroda F., Hiraiwa K., Oshida S. et al., Estimation of postmortem interval from rectal temperature by use of computer (III)-thermal conductivity of the skin. Med. Sci. Law. 1982. 22, No. 4. P. 285-289.

24. F. Brion, B. Marc, F. Launay, Postmortem interval estimation by creatinine levels in human psoas Muscle. Forensic Sci. Int. 1991. 52, No. 1. P. 113 120.

25. Tomita Y., Nihira M., Ohno Y. et al., Histological study of early postmortem changes in various organs: comparison of the paraffin embedding

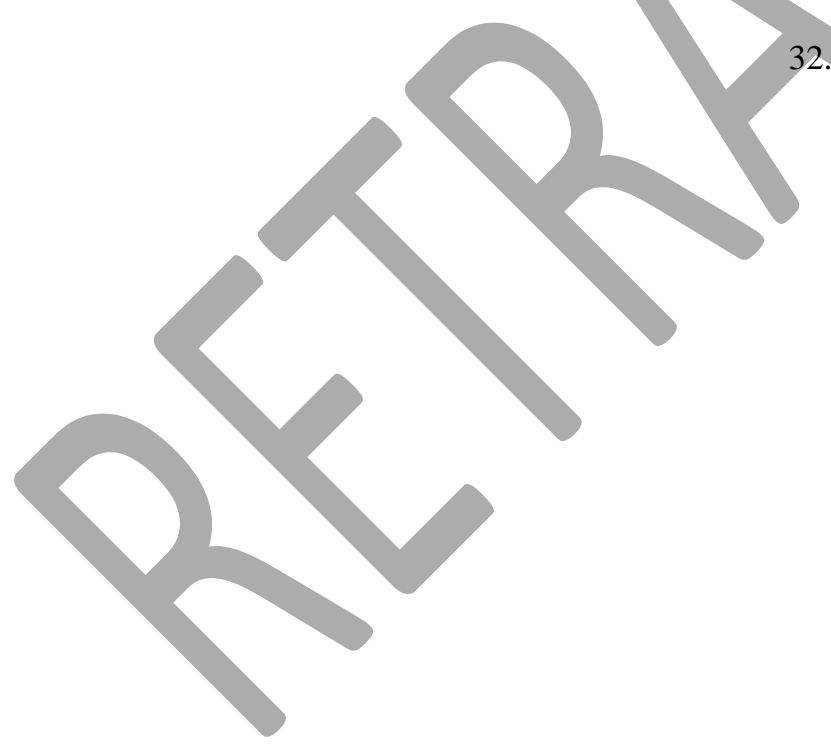

method and the epoxy resin embedding method. Nippon Hoigaku Zasshi. 1999. 53, No. 2. P. 207217.

26. Elmas I., Baslo M.B., Ertas M. et al., Compound muscle action potential analysis in different death models: Significance for the estimation of early postmortem interval. Forensic Sci. Int. 2002. 127, No. 1-2. P. 75-81.

27. Munoz J.I., Suarez-Penaranda J.M., Otero X.L. et al., A new perspective in the estimation of postmortem interval (PMI) based on vitreous. $J$. Forensic Sci. 2001. 46, No. 2. P. 209-214.

28. Goodman J.W. Statistical properties of laser speckle patters. In: Laser Speckle and Related Phenomena, Ed. J.C. Dainty. Berlin, SpringerVerlag, 1975. P. 9-75.

29. Karachevtsev A.O. Fourier Stokes-polarimetry of biological layers polycrystalline networks. Semiconductor Physics, Quantum Electronics \& Optoelectronics. 2012. 15, No. 3.P. 252-268.

30. Gerrard A., Burch J.M. Introduction to Matrix Methods in Optics. A Wiley-Intersci. Publ., New York, 1975.

31. Ushenko Yu.A., Gorsky M.P., Dubolazov A.V., Motrich A.V., Ushenko V.A., Sidor M.I. Spatialfrequency Fourier polarimetry of the complex degree of mutual anisotropy of linear and circular birefringence in the diagnostics of oncological changes in morphological structure of biological tissues. Quantum Electronics. 2012. 42, No. 8. P. 727.

32. Ushenko V.A. Complex degree of mutual coherence of biological liquids, in: ROMOPTO Intern. Conference on Micro- to Nano-Photonics III (P. 88820V-88820V), Intern. Society for Optics and Photonics, 2013. 